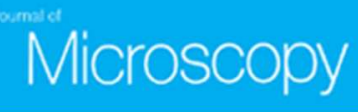

\title{
Techniques for RNA in vivo imaging in plants
}

\begin{tabular}{|r|l|}
\hline Journal: & Journal of Microscopy \\
\hline Manuscript ID: & JMI-2014-0134.R1 \\
\hline Wiley - Manuscript type: & Invited Review \\
\hline Date Submitted by the Author: & n/a \\
\hline Complete List of Authors: & $\begin{array}{l}\text { Tilsner, Jens; University of St Andrews, Biomolecular Sciences Research } \\
\text { Complex }\end{array}$ \\
\hline Keywords: & RNA localisation, molecular beacon, MS2, lambdaN, Pumilio \\
\hline \multicolumn{2}{|l}{} \\
\hline
\end{tabular}

SCHOLARONE ${ }^{\mathrm{m}}$

Manuscripts 


\section{Short Review}

Techniques for RNA in vivo imaging in plants

Jens Tilsner

Biomedical Sciences Research Complex, University of St Andrews, BMS Building, North Haugh, St Andrews, Fife KY 16 9ST, Scotland, U.K.

Cell \& Molecular Science, The James Hutton Institute, Invergowrie, Dundee, DD2 5DA, Scotland, U.K.

Tel. $+44(0) 1334464829$

Fax $+44(0) 1334462595$

jt58@st-andrews.ac.uk

Key words. RNA localisation, molecular beacon, MS2, $\lambda \mathrm{N}$, Pumilio

\section{ABSTRACT}

Since the discovery of small RNAs and RNA silencing, RNA biology has taken a centre stage in cell and developmental biology. Small RNAs, but also mRNAs and other types of cellular and viral RNAs are processed at specific subcellular localisations. To fully understand cellular RNA metabolism and the various processes influenced by it, techniques are required that permit the sequence-specific tracking of RNAs in living cells. A variety of methods for RNA visualisation have been developed since the 1990s, but plant cells pose particular challenges and not all approaches are applicable to them.

On the other hand, plant RNA metabolism is particularly diverse and RNAs are even transported between cells, so RNA imaging can potentially provide many valuable insights into plant function at 
the cellular and tissue level. This Short Review briefly introduces the currently available techniques for plant RNA in vivo imaging and discusses their suitability for different biological questions.

\section{INTRODUCTION}

Many RNAs in cells exhibit dynamic, functional localisations related to processes such as nuclear export, localised translation, RNA turnover within nuclear and cytoplasmic granules, and intercellular communication. Fully understanding the functional significance of these localisations and the mechanisms that underlie them requires the ability to track RNAs sequence-specifically in living cells. A variety of RNA imaging techniques have been developed, but their use in plants, where RNA localisations are still little understood, is limited by factors such as the impenetrability of the cell wall, and sources of auto-fluorescence such as chloroplasts and phenolic cell wall compounds. This Short Review compares live-cell RNA visualisation techniques that have been used in plants, highlighting their advantages and limitations.

\section{APPROACHES BASED ON FLUORESCENTLY LABELLED NUCLEIC ACIDS}

RNA can be fluorescently labelled either by direct incorporation of fluorescent nucleotides, or by hybridisation with a fluorescent probe. Both of these approaches are feasible in vivo but require invasive delivery into cells. The permeabilisation and transfection techniques routinely used in animal cell culture do not work on walled plant cells. In planta, this limits invasive delivery of fluorescent nucleic acids to microprojectile bombardment or micro-injection, the former of which causes significant damage to the cell whereas the latter is technically challenging and extremely lowthroughput. Electroporation or PEG-mediated transformation are only possible in protoplasts, i.e. cells removed from their tissue context and significantly stressed. Nevertheless, these invasive imaging techniques are valuable for plant research because they can enable visualisation of RNAs 
that are unsuitable for genetically encoded sensors, e.g. small RNAs such as siRNAs and miRNAs, which would become non-functional if tagged with additional sequences, or sequestered by RNAbinding proteins.

\subsection{Direct labelling}

RNAs can be rendered fluorescent by incorporation of fluorescent nucleotide derivatives. To do this in a sequence-specific manner, the fluorescent RNA has to be transcribed or synthesized in vitro. If unincorporated fluorescent nucleotides are removed after synthesis, direct labelling allows for essentially background-free RNA imaging.

The main disadvantage of direct labelling is that the invasive introduction of the labelled RNA into the cell bypasses all nuclear and many cytoplasmic processing steps, resulting in potentially significantly altered protein associations compared to endogenous transcripts. The amount of labelled RNA introduced into the cell may also differ significantly from endogenous levels and thus overload cellular processing and localization machineries.

Direct labelling has been used to study the behaviour of uncapped mRNAs and the early events of RNA virus infections in plant cells. Fluorescent, non-capped mRNA PEF-transformed into protoplasts became trapped in the nucleus when nuclear export was inhibited with leptomycin B (Stuger \& Forreiter, 2004). Directly labelled genomic RNA of Tobacco mosaic virus was recruited into endomembrane/cytoskeleton-associated motile granules in a cap-dependent manner (Christensen et al., 2009). Directly end-labelled 21 nucleotide siRNA duplexes were used in leaf bombardment assays to demonstrate that they function as mobile silencing signals between cells (Figure 1A). The subcellular localisations of these small RNAs were not further analysed in these experiments (Dunoyer et al., 2010). 


\subsection{Molecular beacons}

Sequence-specific visualisation of RNAs by fluorescent in situ hybridisation (FISH) is a common technique in animal cell biology, though subcellular resolution is more difficult to achieve in plant samples. Hybridisation-based approaches can also be used in vivo, but because unlike FISH, unbound probe cannot easily be washed out, this requires probes that allow distinguishing between unbound and target-bound forms. Many different design variants have been developed for this purpose (Bao et al., 2009), which are beyond the scope of this Short Review. The only hybridisation-based RNA probes used in live plant cells to date are molecular beacons (MBs). MBs are short hairpin-structured nucleic acids coupled to a fluorophore on one end, and a quencher molecule on the other. In the unbound form, the MB stem-loop brings the fluorophore and quencher into close proximity, preventing fluorescence. Binding of the MB to its target RNA separates the hairpin stems and thus leads to unquenching of the fluorophore.

The major benefit of MBs for live-cell imaging is that they permit visualising endogenously expressed and processed RNAs. Apart from the invasive delivery, their main drawback is that they require extensive optimisation, especially for in vivo uses. The target sequence has to be accessible and not hidden by secondary structures. The free energy of hybridisation to the target has to be sufficient to open the hairpin, but the stem-loop has to be stable enough not to open in the absence of target in vivo. Nonspecific fluorescence can also occur due to endonuclease processing. One way to overcome problems with nonspecific fluorescence is to include a MB with no cellular targets as an internal control for ratiometric imaging. In animal cells, nonspecific nuclear sequestration of beacons often needs to be prevented by linking them to large carriers such as PEG or streptavidin. In plants, 2'-Omethyl-RNA MBs accumulated in the nucleus whereas DNA beacons did not (Göhring et al., 2014) (Figure 1B).

Using MBs, Göhring et al. (2014) were able to distinguish different mRNA splice variants in electroporated Arabidopsis protoplasts. They found that intron-retaining transcripts that were 
insensitive to nonsense-mediated decay (NMD) were retained in the nucleus, thereby evading the cytoplasmic NMD machinery. These splice variants also showed reduced mobility in the nucleus, possibly due to a different ribonucleoprotein complex composition. Subsequently, the authors further optimised their MB protocol and achieved the first single molecule RNA detection in plants.

\section{GENETICALLY ENCODED RNA REPORTERS}

Invasive delivery of RNA probes can be avoided by using genetically encoded reporters, thereby facilitating imaging in a large number of cells within the context of intact tissues. Sequence-specific RNA detection is possible with sequence-specific RNA-binding proteins (RBPs) fused to fluorescent proteins (FPs). Generally applicable methods that can be adapted to any RNA of interest require either tagging of the RNA with an RBP target sequence, or RBPs whose specificity can be predictably modified. Both types of reporters have been used in plants. It is also necessary to distinguish free and RNA-bound RBP-FP fusions. Two different strategies have been employed: nuclear retention of unbound reporter, or fluorescence complementation between two RBP-splitFP fusions binding the same RNA.

\section{1. $M S 2 C P$ and $\lambda N_{22}$}

The capsid protein of bacteriophage MS2 (MS2CP), and a 22-amino acid peptide of the N protein of bacteriophage $\lambda\left(\lambda \mathrm{N}_{22}\right)$ are both RBPs that recognise 19 and $15 \mathrm{Nt}$, stem-loop forming-sequences (MS2 and boxB) with dissociation constants of 6.2 and $22 \mathrm{nM}$, respectively, and have no targets within plant genomes. For RNA imaging, either MS2CP or $\lambda N_{22}$ have been fused to an FP and a nuclear localisation signal. Thus, the fusion proteins are sequestered in the nucleus in the absence of a target RNA. The presence of the target RNA leads to a re-localization of the RNA-reporter into the cytoplasm. (Similar systems were also developed using the BgIG antiterminator protein and the 
bacteriophage PP7 coat protein with their respective corresponding stem-loops, but neither of these systems has so far been applied in plants.)

A downside of the MS2CP or $\lambda N_{22}$ systems is that the RNA of interest has to be tagged with the MS2 or boxB stem-loops, and thus needs to be expressed as a transgene. Preferably the RNA is transcribed from its native promoter, but due to the random insertion of T-DNAs into the genomes of higher plants, its expression level and nuclear processing may still differ from the native transcript, especially if the transgene does not include introns. Additionally, the secondary structure introduced by the MS2 or boxB tags may disrupt function or location of modified RNAs. On the other hand, an advantage of these systems is that very high sensitivities can be achieved by using multiple tandem copies of the stem-loop tags (typically 6-24). In animal systems, a 96xMS2 tag enabled single-molecule-sensitive RNA imaging, but obviously, increasingly large tags with extensive secondary structure exacerbate the risk of disrupting RNA processing and localisation.

MS2CP was the first genetically encoded RNA imaging system described (Bertrand et al. 1998) and is so far the one most extensively used in plants, where its applications have included tracking of storage protein-coding and other mRNAs, viral RNAs, and analysis of nuclear miRNA processing bodies. Between 2 and 24 copies of the MS2 tag, and both nuclear-targeted and cytoplasmic MS2CPGFP fusions were used in these studies. Recently, Schönberger et al. (2012) have developed Gateway-based, 35 S promoter-driven plant expression vectors for tagging of target RNAs with $6 \times M S 2$ or $16 x$ boxB hairpins, respectively, at either the $5^{\prime}$ or the $3^{\prime}$ end. They also constructed expression constructs for multiple spectral variants of nuclear-targeted MS2CP-FP and $\lambda N_{22}-\mathrm{FP}$ fusions. With these systems, they demonstrated the possibility of simultaneously imaging two different RNAs in the same plant cell. Both were full genomic transcripts including UTRs and introns. One RNA encoded a soluble protein, the other a membrane-targeted protein, so their translation should occur in the cytoplasm and on the ER, respectively. Two-colour imaging showed that both were targeted to different transport or processing granules (Figure 1C). Interestingly, in the absence 
of target RNA, both MS2CP-FP and $\lambda \mathrm{N}_{22}-\mathrm{FP}$ fusions accumulated in the RNA-rich environment of the nucleolus despite the absence of natural binding sites in the endogenous transcriptome. The authors also showed that in plants, insertion of MS2 or boxB stem-loops directly upstream of an open reading frame prevented its translation. Thus, tagging downstream of the stop codon is the preferred choice in plants, although in yeast in some cases, $3^{\prime}$ tags have disturbed mRNA localisations whereas $5^{\prime}$ tags did not.

\subsection{Pumilio RNA binding domain}

Pumilio/FBF family (PUF) proteins are sequence-specific RBPs found in all eukaryotes (26 in Arabidopsis). Their RNA binding domain, the Pumilio homology domain (PUMHD) consists of eight tandem repeat Puf motifs that each bind one nucleotide in an $8 \mathrm{Nt}$ target sequence. Sequencespecific interactions are mediated by the side chains of two amino acids per Puf repeat and the RNA nucleobases (a third amino acid side chain in each repeat forms a non-specific stacking interaction with the RNA). This makes it possible to re-engineer the specificity of the PUMHD with relatively few modifications. Structural analysis of the native human Pumilio 1 and molecular evolution have produced a complete code for recognition of the four RNA bases, and a GoldenGate pipeline for rapid assembly of any PUMHD variant has been developed (Abil et al., 2014). Within certain limitations (which are beyond the scope of this Short Review), the PUMHD can thus be engineered to bind any RNA of choice. When used for RNA imaging, this means that in contrast to MS2CP and $\lambda N_{22}$ systems, untagged, native RNAs can be imaged. Alternatively, RNAs can be tagged with recognition motifs of selected PUMHD variants. The benefit in this case is that these tags do not introduce stable secondary structures. Wild-type PUMHD of human Pumilio 1 also has a very high affinity to its target sequence $\left(k_{D}=0.48 \mathrm{nM}\right)$, and variants with a $k_{D}$ as low as $0.05 \mathrm{nM}$ have been described. This makes PUMHD-based RNA imaging potentially more sensitive than MS2CP- or $\lambda \mathrm{N}_{22}-$ FP fusions, and single molecule imaging has been achieved in animal cells. On the other hand, the 
RNA affinity of PUMHD variants modified to bind novel RNA sequences is still unpredictable and can be an order of magnitude lower than the wild-type protein.

Disadvantages of the PUMHD compared with MS2CP and $\lambda \mathrm{N}_{22}$ are the short length of the $8 \mathrm{Nt}$ target sequence and the occurrence of natural binding sites in endogenous plant mRNAs. Additionally, the PUMHD shows degrees of binding preference for target sequence variants, rather than complete specificity. Because off-target binding in a complex eukaryotic cell in vivo is thus practically unavoidable, combinations of two PUMHDs are used to increase the specificity of RNA imaging. To distinguish RNA-bound and free reporter constructs, the two PUMHD variants are coupled to the two halves of a split FP for bimolecular fluorescence complementation (BiFC). The two modified PUMHDs bind closely adjacent (5-9 Nt) binding sites on the same RNA, so that fluorescence reconstitution occurs upon binding of both split FP fusion proteins to the same RNA. PUMHD-BiFC is unfortunately not background free, as the reconstituted FP is extremely stable and the fluorescent complex accumulates in plant cells. In animal cells, nuclear-targeted fusions of two PUMHDs coupled to a FP have also been used to distinguish free and RNA-bound fluorescence similar to the MS2CP and $\lambda N_{22}$ systems, but this approach has yet to be tested in plants.

In plants, PUMHD-BiFC imaging has been used to track vial RNAs (Tilsner et al., 2009). This has enabled the observations that potyvirus replication complexes develop from ER-associated to chloroplast-associated membrane sites, and that replication and cell-to-cell movement of a potexvirus are spatially coupled at plasmodesmata (Figure 1D).

\section{CHOICE OF REPORTER}

This Short Review has described RNA in vivo imaging systems that have been successfully used in plants, and compared their respective advantages and drawbacks (Table 1), as well as highlighting some insights obtained with each. No single technique suits every experimental system, and the 
choice of RNA reporter must depend on the type of RNA that is being studied and the types of questions that need to be addressed. For small RNAs direct labelling or molecular beacons are probably the only suitable in vivo approaches. For mRNA imaging, considerations need to include how abundant these are and which RNA processing pathways need to remain unaffected by the imaging system, as well as if imaging can be performed in protoplasts or requires intact tissue. The main consideration for imaging viral RNAs is how tags and RBPs affect their infectivity. As RNA imaging systems are being developed further, the available toolbox will become even more diverse. Some promising reporters, such as RNA aptamer tags like "Spinach" (and its improved derivatives "Spinach2" and "BabySpinach"), "Mango" and IMAGE that selectively bind cell-permeant fluorophores, have yet to be adapted to use in plants (Paige et al., 2011). Thus, it is to be hoped that in the near future, studying functional localisations of plant RNAs will become a routine approach.

\section{References}

Abil, Z., Denard, C.A. \& Zhao, H. (2014) Modular assembly of designer PUF proteins for specific post-transcriptional regulation of endogenous RNA. J. Biol. Eng. 8, 7. doi:10.1186/1754-1611-8-7

Bao, G., Rhee, W.J. \& Tsourkas, A. (2009) Fluorescent probes for live-cell RNA detection. Annu. Rev. Bioeng. 11, 25-47. doi: 10.1146/annurev-bioeng-061008-124920

Bertrand, E., Chartrand, P., Schaefer, M., Shenoy, S.M., Singer, R.H. \& Long, R.M. (1998) Localization of ASH1 mRNA particles in living yeast. Mol. Cell 2, 437-445. doi: 10.1016/S10972765(00)80143-4 
Christensen, N., Tilsner, J., Bell, K., Hammann, P., Parton, R., Lacomme, C. \& Oparka, K.J. (2009)

The 5' cap of Tobacco mosaic virus (TMV) is required for virion attachment to the actin/endoplasmic reticulum network during early infection. Traffic 10, 536-551. doi:

10.1111/j.1600-0854.2009.00889.x

Dunoyer, P., Schott, G., Himber, C., Meyer, D., Takeda, A., Carrington, J.C. \& Voinnet, O. (2010) Small RNA duplexes function as mobile silencing signals between plant cells. Science 328, 912916. doi: $10.1126 /$ science. 1185880

Göhring, J., Jacak, J. \& Barta, A. (2014) Imaging of endogenous messenger RNA splice variants in living cells reveals nuclear retention of transcripts inaccessible to nonsense-mediated decay in Arabidopsis. Plant Cell 26, 754-764. doi: 10.1105/tpc.113.118075

Paige, J.S., Wu, K.Y. \& Jaffrey, S.R. (2011) RNA mimics of green fluorescent protein. Science 333, 642-646. doi: 10.1126/science.1207339

Schönberger, J., Hammes, U.Z. \& Dresselhaus, T. (2012) In vivo visualization of RNA in plants cells using the $\lambda N_{22}$ system and a GATEWAY-compatible vector series for candidate RNAs. Plant J. 71, 173-181. doi: 10.1111/j.1365-313X.2012.04923.x

Stuger, R. \& Forreiter, C. (2004) Uncapped mRNA introduced into tobacco protoplasts can be imported into the nucleus and is trapped by leptomycin B. Plant Cell Rep. 23, 99-103. doi: $10.1007 / \mathrm{s} 00299-004-0780-4$ 
Tilsner, J., Linnik, O., Christensen, N.M., Bell, K., Roberts, I.M., Lacomme, C. \& Oparka, K.J. (2009) Live-cell imaging of viral RNA genomes using a Pumilio-based reporter. Plant J. 57, 758-770. doi: 10.1111/j.1365-313X.2008.03720.x

\section{Figure legends}

Fig. 1. Examples of RNA imaging in plant cells.

(A) Direct labelling. Fluorescently end-labelled, double-stranded siRNAs bombarded into an Arabidopsis epidermal leaf cell have moved into surrounding cells (1.) and there caused silencing of a GFP transgene (2.) (Dunoyer et al., 2010). (B) Molecular Beacons. A gene-specific (1.) and a control beacon with no cellular target (2.) were electroporated into an Arabidopsis protoplast (3.). A ratiometric image (4.) is generated representing the signal/noise ratio in each pixel. From this, a cell compartment-specific distribution of background-corrected signal intensities can be averaged for a population of cells (Göhring et al., 2014). (C) MS2CP-CFP and $\lambda \mathrm{N}_{22}$-mVenus. Nuclear-targeted MS2CP-CFP (1.) and $\lambda N_{22}$-mVenus (2.) are both recruited into cytoplasmic granules by their coexpressed target mRNAs tagged with 6xMS2- and 16xboxB, respectively. The MS2CP-imaged mRNA, encoding a cytoplasmic protein, and the $\lambda N_{22}$-imaged RNA, encoding a membrane protein, localise to different granules (Schönberger et al., 2012). (D) PUMHD-BiFC. Potato virus X RNA (green) imaged by 
PUMHD-BiFC (Tilsner et al., 2009) localises to small membrane structures at the entrances of plasmodesmata, which are labelled by the viral capsid protein (red) (Tilsner, unpublished images). 
Table 1

Properties of RNA in vivo imaging systems used in plants.

\begin{tabular}{|c|c|c|}
\hline Technique & Advantages & Disadvantages \\
\hline Direct labelling & $\begin{array}{l}\text { - } \quad \text { No alterations to RNA sequence } \\
\text { - } \quad \text { Very high signal/noise ratio } \\
\text { - } \quad \text { Can use non-natural/modified } \\
\text { RNAs } \\
\text { - Can visualise small RNAs }\end{array}$ & $\begin{array}{l}\text { - Invasive delivery } \\
\text { - } \quad \text { No endogenous processing } \\
\text { - } \quad \text { No native RNA levels }\end{array}$ \\
\hline Molecular Beacons & $\begin{array}{l}\text { - No alterations to RNA sequence } \\
\text { - Native RNA levels and } \\
\text { endogenous processing } \\
\text { - Can visualise small RNAs }\end{array}$ & $\begin{array}{l}\text { - } \quad \text { Invasive delivery } \\
\text { - } \quad \text { Extensive optimisation }\end{array}$ \\
\hline MS2CP-FP \& $\lambda N_{22}-F P$ & $\begin{array}{l}\text { - Non-invasive, easy to use } \\
\text { - Can achieve very high sensitivity } \\
\text { (single molecule) }\end{array}$ & $\begin{array}{l}\text { - RNA tagged, hairpins may } \\
\text { interfere with native localisation } \\
\text { or processing }\end{array}$ \\
\hline PUMHD-BiFC & $\begin{array}{l}\text { - } \quad \text { Non-invasive, easy to use } \\
\text { - } \quad \text { ro alterations to RNA sequence } \\
\text { required } \\
\text { - Native RNA levels and } \\
\text { endogenous processing possible } \\
\text { - Can achieve very high sensitivity } \\
\text { (single molecule) }\end{array}$ & $\begin{array}{l}\text { Problems with nonspecific BiFC } \\
\text { signal }\end{array}$ \\
\hline
\end{tabular}



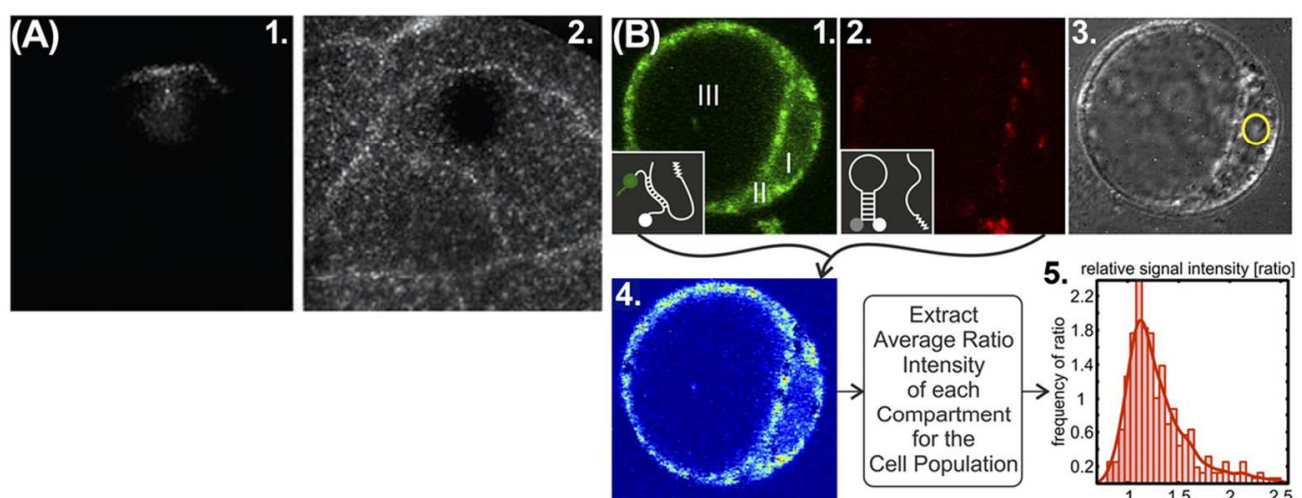

5. relative signal intensity [ratio]
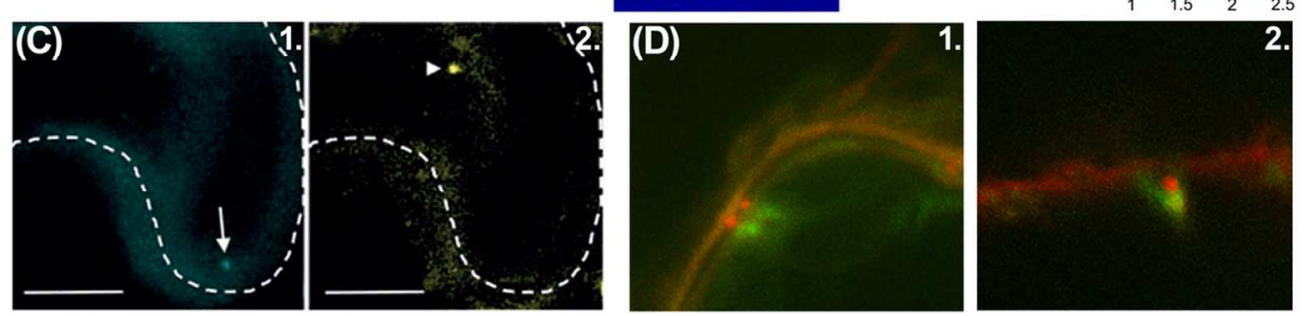

$105 \times 65 \mathrm{~mm}(300 \times 300$ DPI $)$ 


\section{Short Review}

Techniques for RNA in vivo imaging in plants

Jens Tilsner

Biomedical Sciences Research Complex, University of St Andrews, BMS Building, North Haugh, St Andrews, Fife KY 16 9ST, Scotland, U.K.

Cell \& Molecular Science, The James Hutton Institute, Invergowrie, Dundee, DD2 5DA, Scotland, U.K.

Tel. $+44(0) 1334464829$

Fax $+44(0) 1334462595$

jt58@st-andrews.ac.uk

Key words. RNA localisation, molecular beacon, MS2, $\lambda \mathrm{N}$, Pumilio

\section{ABSTRACT}

Since the discovery of small RNAs and RNA silencing, RNA biology has taken a centre stage in cell and developmental biology. Small RNAs, but also mRNAs and other types of cellular and viral RNAs are processed at specific subcellular localisations. To fully understand cellular RNA metabolism and the various processes influenced by it, techniques are required that permit the sequence-specific tracking of RNAs in living cells. A variety of methods for RNA visualisation have been developed since the 1990s, but plant cells pose particular challenges and not all approaches are applicable to them.

On the other hand, plant RNA metabolism is particularly diverse and RNAs are even transported between cells, so RNA imaging can potentially provide many valuable insights into plant function at 
the cellular and tissue level. This Short Review briefly introduces the currently available techniques for plant RNA in vivo imaging and discusses their suitability for different biological questions.

\section{INTRODUCTION}

Many RNAs in cells exhibit dynamic, functional localisations related to processes such as nuclear export, localised translation, RNA turnover within nuclear and cytoplasmic granules, and intercellular communication. Fully understanding the functional significance of these localisations and the mechanisms that underlie them requires the ability to track RNAs sequence-specifically in living cells. A variety of RNA imaging techniques have been developed, but their use in plants, where RNA localisations are still little understood, is limited by factors such as the impenetrability of the cell wall, and sources of auto-fluorescence such as chloroplasts and phenolic cell wall compounds. This Short Review compares live-cell RNA visualisation techniques that have been used in plants, highlighting their advantages and limitations.

\section{APPROACHES BASED ON FLUORESCENTLY LABELLED NUCLEIC ACIDS}

RNA can be fluorescently labelled either by direct incorporation of fluorescent nucleotides, or by hybridisation with a fluorescent probe. Both of these approaches are feasible in vivo but require invasive delivery into cells. The permeabilisation and transfection techniques routinely used in animal cell culture do not work on walled plant cells. In planta, this limits invasive delivery of fluorescent nucleic acids to microprojectile bombardment or micro-injection, the former of which causes significant damage to the cell whereas the latter is technically challenging and extremely lowthroughput. Electroporation or PEG-mediated transformation are only possible in protoplasts, i.e. cells removed from their tissue context and significantly stressed. Nevertheless, these invasive imaging techniques are valuable for plant research because they can enable visualisation of RNAs 
that are unsuitable for genetically encoded sensors, e.g. small RNAs such as siRNAs and miRNAs, which would become non-functional if tagged with additional sequences, or sequestered by RNAbinding proteins.

\subsection{Direct labelling}

RNAs can be rendered fluorescent by incorporation of fluorescent nucleotide derivatives. To do this in a sequence-specific manner, the fluorescent RNA has to be transcribed or synthesized in vitro. If unincorporated fluorescent nucleotides are removed after synthesis, direct labelling allows for essentially background-free RNA imaging.

The main disadvantage of direct labelling is that the invasive introduction of the labelled RNA into the cell bypasses all nuclear and many cytoplasmic processing steps, resulting in potentially significantly altered protein associations compared to endogenous transcripts. The amount of labelled RNA introduced into the cell may also differ significantly from endogenous levels and thus overload cellular processing and localization machineries.

Direct labelling has been used to study the behaviour of uncapped mRNAs and the early events of RNA virus infections in plant cells. Fluorescent, non-capped mRNA PEF-transformed into protoplasts became trapped in the nucleus when nuclear export was inhibited with leptomycin B (Stuger \& Forreiter, 2004). Directly labelled genomic RNA of Tobacco mosaic virus was recruited into endomembrane/cytoskeleton-associated motile granules in a cap-dependent manner (Christensen et al., 2009). Directly end-labelled 21 nucleotide siRNA duplexes were used in leaf bombardment assays to demonstrate that they function as mobile silencing signals between cells (Figure 1A). The subcellular localisations of these small RNAs were not further analysed in these experiments (Dunoyer et al., 2010). 


\subsection{Molecular beacons}

Sequence-specific visualisation of RNAs by fluorescent in situ hybridisation (FISH) is a common technique in animal cell biology, though subcellular resolution is more difficult to achieve in plant samples. Hybridisation-based approaches can also be used in vivo, but because unlike FISH, unbound probe cannot easily be washed out, this requires probes that allow distinguishing between unbound and target-bound forms. Many different design variants have been developed for this purpose (Bao et al., 2009), which are beyond the scope of this Short Review. The only hybridisation-based RNA probes used in live plant cells to date are molecular beacons (MBs). MBs are short hairpin-structured nucleic acids coupled to a fluorophore on one end, and a quencher molecule on the other. In the unbound form, the MB stem-loop brings the fluorophore and quencher into close proximity, preventing fluorescence. Binding of the MB to its target RNA separates the hairpin stems and thus leads to unquenching of the fluorophore.

The major benefit of MBs for live-cell imaging is that they permit visualising endogenously expressed and processed RNAs. Apart from the invasive delivery, their main drawback is that they require extensive optimisation, especially for in vivo uses. The target sequence has to be accessible and not hidden by secondary structures. The free energy of hybridisation to the target has to be sufficient to open the hairpin, but the stem-loop has to be stable enough not to open in the absence of target in vivo. Nonspecific fluorescence can also occur due to endonuclease processing. One way to overcome problems with nonspecific fluorescence is to include a MB with no cellular targets as an internal control for ratiometric imaging. In animal cells, nonspecific nuclear sequestration of beacons often needs to be prevented by linking them to large carriers such as PEG or streptavidin. In plants, 2'-Omethyl-RNA MBs accumulated in the nucleus whereas DNA beacons did not (Göhring et al., 2014) (Figure 1B).

In the only study so faruU Using MBsin plants, Göhring et al. (2014) were able to distinguish different mRNA splice variants in electroporated Arabidopsis protoplasts. They found that intron-retaining 
transcripts that were insensitive to nonsense-mediated decay (NMD) were retained in the nucleus, thereby evading the cytoplasmic NMD machinery. These splice variants also showed reduced mobility in the nucleus, possibly due to a different ribonucleoprotein complex composition. Subsequently, the authors further optimised their MB protocol and achieved the first single molecule RNA detection in plants.

\section{GENETICALLY ENCODED RNA REPORTERS}

Invasive delivery of RNA probes can be avoided by using genetically encoded reporters, thereby facilitating imaging in a large number of cells within the context of intact tissues. Sequence-specific RNA detection is possible with sequence-specific RNA-binding proteins (RBPs) fused to fluorescent proteins (FPs). Generally applicable methods that can be adapted to any RNA of interest require either tagging of the RNA with an RBP target sequence, or RBPs whose specificity can be predictably modified. Both types of reporters have been used in plants. It is also necessary to distinguish free and RNA-bound RBP-FP fusions. Two different strategies have been employed: nuclear retention of unbound reporter, or fluorescence complementation between two RBP-splitFP fusions binding the same RNA.

\section{1. $M S 2 C P$ and $\lambda N_{22}$}

The capsid protein of bacteriophage MS2 (MS2CP), and a 22-amino acid peptide of the N protein of bacteriophage $\lambda\left(\lambda \mathrm{N}_{22}\right)$ are both RBPs that recognise 19 and $15 \mathrm{Nt}$, stem-loop forming-sequences (MS2 and boxB) with dissociation constants of 6.2 and $22 \mathrm{nM}$, respectively, and have no targets within plant genomes. For RNA imaging, either MS2CP or $\lambda N_{22}$ have been fused to an FP and a nuclear localisation signal. Thus, the fusion proteins are sequestered in the nucleus in the absence of a target RNA. The presence of the target RNA leads to a re-localization of the RNA-reporter into the cytoplasm. (Similar systems were also developed using the BglG antiterminator protein and the 
bacteriophage PP7 coat protein with their respective corresponding stem-loops, but neither of these systems has so far been applied in plants.)

A downside of the MS2CP or $\lambda N_{22}$ systems is that the RNA of interest has to be tagged with the MS2 or boxB stem-loops, and thus needs to be expressed as a transgene. Preferably the RNA is transcribed from its native promoter, but due to the random insertion of T-DNAs into the genomes of higher plants, its expression level and nuclear processing may still differ from the native transcript, especially if the transgene does not include introns. Additionally, the secondary structure introduced by the MS2 or boxB tags may disrupt function or location of modified RNAs. On the other hand, an advantage of these systems is that very high sensitivities can be achieved by using multiple tandem copies of the stem-loop tags (typically 6-24). In animal systems, a 96xMS2 tag enabled single-molecule-sensitive RNA imaging, but obviously, increasingly large tags with extensive secondary structure exacerbate the risk of disrupting RNA processing and localisation.

MS2CP was the first genetically encoded RNA imaging system described (Bertrand et al. 1998) and is so far the one most extensively used in plants, where its applications have included tracking of storage protein-coding and other mRNAs, viral RNAs, and analysis of nuclear miRNA processing bodies. Between 2 and 24 copies of the MS2 tag, and both nuclear-targeted and cytoplasmic MS2CPGFP fusions were used in these studies. Recently, Schönberger et al. (2012) have developed Gateway-based, 35 S promoter-driven plant expression vectors for tagging of target RNAs with $6 \times M S 2$ or $16 x$ boxB hairpins, respectively, at either the $5^{\prime}$ or the $3^{\prime}$ end. They also constructed expression constructs for multiple spectral variants of nuclear-targeted MS2CP-FP and $\lambda \mathrm{N}_{22}-\mathrm{FP}$ fusions. With these systems, they demonstrated the possibility of simultaneously imaging two different RNAs in the same plant cell. Both were full genomic transcripts including UTRs and introns. One RNA encoded a soluble protein, the other a membrane-targeted protein, so their translation should occur in the cytoplasm and on the ER, respectively. Two-colour imaging showed that both were targeted to different transport or processing granules (Figure 1C). Interestingly, in the absence 
of target RNA, both MS2CP-FP and $\lambda \mathrm{N}_{22}-\mathrm{FP}$ fusions accumulated in the RNA-rich environment of the nucleolus despite the absence of natural binding sites in the endogenous transcriptome. The authors also showed that in plants, insertion of MS2 or boxB stem-loops directly upstream of an open reading frame prevented its translation. Thus, tagging downstream of the stop codon is the preferred choice in plants, although in yeast in some cases, $3^{\prime}$ tags have disturbed mRNA localisations whereas $5^{\prime}$ tags did not.

\subsection{Pumilio RNA binding domain}

Pumilio/FBF family (PUF) proteins are sequence-specific RBPs found in all eukaryotes (26 in Arabidopsis). Their RNA binding domain, the Pumilio homology domain (PUMHD) consists of eight tandem repeat Puf motifs that each bind one nucleotide in an $8 \mathrm{Nt}$ target sequence. Sequencespecific interactions are mediated by the side chains of two amino acids per Puf repeat and the RNA nucleobases (a third amino acid side chain in each repeat forms a non-specific stacking interaction with the RNA). This makes it possible to re-engineer the specificity of the PUMHD with relatively few modifications. Structural analysis of the native human Pumilio 1 and molecular evolution have produced a complete code for recognition of the four RNA bases, and a GoldenGate pipeline for rapid assembly of any PUMHD variant has been developed (Abil et al., 2014). Within certain limitations (which are beyond the scope of this Short Review), the PUMHD can thus be engineered to bind any RNA of choice. When used for RNA imaging, this means that in contrast to MS2CP and $\lambda N_{22}$ systems, untagged, native RNAs can be imaged. Alternatively, RNAs can be tagged with recognition motifs of selected PUMHD variants. The benefit in this case is that these tags do not introduce stable secondary structures. Wild-type PUMHD of human Pumilio 1 also has a very high affinity to its target sequence $\left(k_{D}=0.48 \mathrm{nM}\right)$, and variants with a $k_{D}$ as low as $0.05 \mathrm{nM}$ have been described. This makes PUMHD-based RNA imaging potentially more sensitive than MS2CP- or $\lambda \mathrm{N}_{22}-$ FP fusions, and single molecule imaging has been achieved in animal cells. On the other hand, the 
RNA affinity of PUMHD variants modified to bind novel RNA sequences is still unpredictable and can be an order of magnitude lower than the wild-type protein.

Disadvantages of the PUMHD compared with MS2CP and $\lambda \mathrm{N}_{22}$ are the short length of the $8 \mathrm{Nt}$ target sequence and the occurrence of natural binding sites in endogenous plant mRNAs. Additionally, the PUMHD shows degrees of binding preference for target sequence variants, rather than complete specificity. Because off-target binding in a complex eukaryotic cell in vivo is thus practically unavoidable, combinations of two PUMHDs are used to increase the specificity of RNA imaging. To distinguish RNA-bound and free reporter constructs, the two PUMHD variants are coupled to the two halves of a split FP for bimolecular fluorescence complementation (BiFC). The two modified PUMHDs bind closely adjacent (5-9 Nt) binding sites on the same RNA, so that fluorescence reconstitution occurs upon binding of both split FP fusion proteins to the same RNA. PUMHD-BiFC is unfortunately not background free, as the reconstituted FP is extremely stable and the fluorescent complex accumulates in plant cells. In animal cells, nuclear-targeted fusions of two PUMHDs coupled to a FP have also been used to distinguish free and RNA-bound fluorescence similar to the MS2CP and $\lambda N_{22}$ systems, but this approach has yet to be tested in plants.

In plants, PUMHD-BiFC imaging has been used to track vial RNAs (Tilsner et al., 2009). This has enabled the observations that potyvirus replication complexes develop from ER-associated to chloroplast-associated membrane sites, and that replication and cell-to-cell movement of a potexvirus are spatially coupled at plasmodesmata (Figure 1D).

\section{CHOICE OF REPORTER}

This Short Review has described RNA in vivo imaging systems that have been successfully used in plants, and compared their respective advantages and drawbacks (Table 1), as well as highlighting some insights obtained with each. No single technique suits every experimental system, and the 
choice of RNA reporter must depend on the type of RNA that is being studied and the types of questions that need to be addressed. For small RNAs direct labelling or molecular beacons are probably the only suitable in vivo approaches. For mRNA imaging, considerations need to include how abundant these are and which RNA processing pathways need to remain unaffected by the imaging system, as well as if imaging can be performed in protoplasts or requires intact tissue. The main consideration for imaging viral RNAs is how tags and RBPs affect their infectivity. As RNA imaging systems are being developed further, the available toolbox will become even more diverse. Some promising reporters, such as RNA aptamer tags like "Spinach" (and its improved derivatives "Spinach2" and "BabySpinach"), "Mango" and IMAGE that selectively bind cell-permeant fluorophores, have yet to be adapted to use in plants (Paige et al., 2011). Thus, and it is to be hoped that in the near future, studying functional localisations of plant RNAs tocalisations in plants-will become a routine approach in the near future.

\section{References}

Abil, Z., Denard, C.A. \& Zhao, H. (2014) Modular assembly of designer PUF proteins for specific post-transcriptional regulation of endogenous RNA. J. Biol. Eng. 8, 7. doi:10.1186/1754-1611-8-7

Bao, G., Rhee, W.J. \& Tsourkas, A. (2009) Fluorescent probes for live-cell RNA detection. Annu. Rev. Bioeng. 11, 25-47. doi: 10.1146/annurev-bioeng-061008-124920

Bertrand, E., Chartrand, P., Schaefer, M., Shenoy, S.M., Singer, R.H. \& Long, R.M. (1998) Localization of ASH1 mRNA particles in living yeast. Mol. Cell 2, 437-445. doi: 10.1016/S10972765(00)80143-4 
Christensen, N., Tilsner, J., Bell, K., Hammann, P., Parton, R., Lacomme, C. \& Oparka, K.J. (2009)

The 5' cap of Tobacco mosaic virus (TMV) is required for virion attachment to the actin/endoplasmic reticulum network during early infection. Traffic 10, 536-551. doi:

10.1111/j.1600-0854.2009.00889.x

Dunoyer, P., Schott, G., Himber, C., Meyer, D., Takeda, A., Carrington, J.C. \& Voinnet, O. (2010) Small RNA duplexes function as mobile silencing signals between plant cells. Science 328, 912916. doi: $10.1126 /$ science. 1185880

Göhring, J., Jacak, J. \& Barta, A. (2014) Imaging of endogenous messenger RNA splice variants in living cells reveals nuclear retention of transcripts inaccessible to nonsense-mediated decay in Arabidopsis. Plant Cell 26, 754-764. doi: 10.1105/tpc.113.118075

Paige, J.S., Wu, K.Y. \& Jaffrey, S.R. (2011) RNA mimics of green fluorescent protein. Science 333, 642-646. doi: 10.1126/science.1207339

Schönberger, J., Hammes, U.Z. \& Dresselhaus, T. (2012) In vivo visualization of RNA in plants cells using the $\lambda \mathrm{N}_{22}$ system and a GATEWAY-compatible vector series for candidate RNAs. Plant J. 71, 173-181. doi: 10.1111/j.1365-313X.2012.04923.x

Stuger, R. \& Forreiter, C. (2004) Uncapped mRNA introduced into tobacco protoplasts can be imported into the nucleus and is trapped by leptomycin B. Plant Cell Rep. 23, 99-103. doi: $10.1007 / \mathrm{s} 00299-004-0780-4$ 
Tilsner, J., Linnik, O., Christensen, N.M., Bell, K., Roberts, I.M., Lacomme, C. \& Oparka, K.J. (2009) Live-cell imaging of viral RNA genomes using a Pumilio-based reporter. Plant J. 57, 758-770. doi: 10.1111/j.1365-313X.2008.03720.x

\section{Figure legends}

Fig. 1. Examples of RNA imaging in plant cells.

(A) Direct labelling. Fluorescently end-labelled, double-stranded siRNAs bombarded into an Arabidopsis epidermal leaf cell have moved into surrounding cells (1.) and there caused silencing of a GFP transgene (2.) (Dunoyer et al., 2010). (B) Molecular Beacons. A gene-specific (1.) and a control beacon with no cellular target (2.) were electroporated into an Arabidopsis protoplast (3.). A ratiometric image (4.) is generated representing the signal/noise ratio in each pixel. From this, a cell compartment-specific distribution of background-corrected signal intensities can be averaged for a population of cells (Göhring et al., 2014). (C) MS2CP-CFP and $\lambda N_{22}$-mVenus. Nuclear-targeted MS2CP-CFP (1.) and $\lambda N_{22}$-mVenus (2.) are both recruited into cytoplasmic granules by their coexpressed target mRNAs tagged with 6xMS2- and 16xboxB, respectively. The MS2CP-imaged mRNA, encoding a cytoplasmic protein, and the $\lambda N_{22}$-imaged RNA, encoding a membrane protein, localise to different granules (Schönberger et al., 2012). (D) PUMHD-BiFC. Potato virus X RNA (green) imaged by 
PUMHD-BiFC (Tilsner et al., 2009) localises to small membrane structures at the entrances of plasmodesmata, which are labelled by the viral capsid protein (red) (Tilsner, unpublished images). 